\title{
Are Generalized and Transverse Momentum Dependent Parton Distributions Related?
}

\author{
Stephan Meißner, Andreas Metz, and Klaus Goeke \\ Institut für Theoretische Physik II, Ruhr-Universität Bochum, \\ D-44780 Bochum, Germany
}

\begin{abstract}
The present knowledge on non-trivial relations between generalized parton distributions on the one hand and transverse momentum dependent distributions on the other is reviewed. While various relations can be found in the framework of spectator models, so far no model-independent non-trivial relations have been established.
\end{abstract}

\section{Definitions and trivial relations}

During the last decade a lot of effort has been devoted to study in detail generalized parton distributions (GPDs) as well as transverse momentum dependent parton distributions (TMDs). While GPDs enter the QCD description of hard exclusive reactions on the nucleon, TMDs appear in connection with various semi-inclusive processes. Recent work has suggested for the first time very interesting non-trivial relations between these two types of parton distributions [1, 2, 3, 4, 5, 6, 7]. The present short note [8] is based on Ref. [7], where the current knowledge on such relations has been reviewed and previous work on this topic has been considerably extended.

To be specific now, two leading twist quark GPDs of the nucleon are defined through

$$
\begin{aligned}
F^{q}\left(x, \Delta ; \lambda, \lambda^{\prime}\right) & =\left.\frac{1}{2} \int \frac{d z^{-}}{2 \pi} e^{i k \cdot z}\left\langle p^{\prime} ; \lambda^{\prime}\left|\bar{\psi}\left(-\frac{1}{2} z\right) \gamma^{+} \mathcal{W}_{\mathrm{GPD}} \psi\left(\frac{1}{2} z\right)\right| p ; \lambda\right\rangle\right|_{z^{+}=\vec{z}_{T}=0} \\
& =\frac{1}{2 P^{+}} \bar{u}\left(p^{\prime}, \lambda^{\prime}\right)\left(\gamma^{+} H^{q}(x, \xi, t)+\frac{i \sigma^{+\mu} \Delta_{\mu}}{2 M} E^{q}(x, \xi, t)\right) u(p, \lambda),
\end{aligned}
$$

with $P=\left(p+p^{\prime}\right) / 2$ denoting the average nucleon momentum and $\Delta=p^{\prime}-p$ the momentum transfer to the nucleon. The GPDs $H^{q}$ and $E^{q}$ depend on the variables

$$
x=\frac{k^{+}}{P^{+}}, \quad \xi=-\frac{\Delta^{+}}{2 P^{+}}, \quad t=\Delta^{2},
$$

where the dependence on the renormalization scale has been suppressed. Note that the Wilson line $\mathcal{W}_{\text {GPD }}$ ensures the color gauge invariance of the bilocal quark operator in (1). The remaining six leading quark GPDs are obtained if one replaces the matrix $\gamma^{+}$in the operator in (1) by $\gamma^{+} \gamma_{5}$ or $i \sigma^{j+} \gamma_{5}$ ( $j$ being a transverse index).

In a similar way, two leading twist quark TMDs are defined according to

$$
\begin{aligned}
\Phi^{q}\left(x, \vec{k}_{T} ; S\right) & =\left.\frac{1}{2} \int \frac{d z^{-}}{2 \pi} \frac{d^{2} \vec{z}_{T}}{(2 \pi)^{2}} e^{i k \cdot z}\left\langle P ; S\left|\bar{\psi}\left(-\frac{1}{2} z\right) \gamma^{+} \mathcal{W}_{\mathrm{TMD}} \psi\left(\frac{1}{2} z\right)\right| P ; S\right\rangle\right|_{z^{+}=0} \\
& =f_{1}^{q}\left(x, \vec{k}_{T}^{2}\right)-\frac{\epsilon_{T}^{i j} k_{T}^{i} S_{T}^{j}}{M} f_{1 T}^{\perp q}\left(x, \vec{k}_{T}^{2}\right)
\end{aligned}
$$

The TMDs depend both on the longitudinal momentum fraction $x$ of the partons and on the transverse parton momentum $\vec{k}_{T}$. While $f_{1}$ is the familiar unpolarized quark distribution, 
$f_{1 T}^{\perp}$ represents the so-called Sivers function [9, 10], which appears for a transversely polarized target and is supposed to be at the origin of various observed single spin phenomena in hard semi-inclusive reactions.

There exist some trivial relations between GPDs and TMDs because of the connection between GPDs (for $\xi=t=0$ ) and TMDs (integrated upon $\vec{k}_{T}$ ) on the one hand and ordinary parton distributions on the other. An example is given by

$$
H^{q}(x, 0,0)=f_{1}^{q}(x)=\int d^{2} \vec{k}_{T} f_{1}^{q}\left(x, \vec{k}_{T}^{2}\right) .
$$

Two additional trivial relations hold on the quark sector (involving the quark helicity and transversity distribution) and also two for gluon distributions. In this note, however, we are mainly interested in non-trivial relations between GPDs and TMDs.

\section{Impact parameter representation of GPDs}

In Ref. [1], a non-trivial relation was proposed for the first time - a connection between the GPD $E$ and the Sivers function $f_{1 T}^{\perp}$. In that work an important role is played by the impact parameter representation of GPDs. For $\xi=0$, GPDs in impact parameter space have a density interpretation, and are generically given by

$$
\mathcal{X}\left(x, \vec{b}_{T}^{2}\right)=\int \frac{d^{2} \vec{\Delta}_{T}}{(2 \pi)^{2}} e^{-i \vec{\Delta}_{T} \cdot \vec{b}_{T}} X\left(x, 0,-\vec{\Delta}_{T}^{2}\right) .
$$

Using this definition, the Fourier transform of the correlator in (1) (for $\xi=0$ ) has the form

$$
\mathcal{F}^{q}\left(x, \vec{b}_{T} ; S\right)=\int \frac{d^{2} \vec{\Delta}_{T}}{(2 \pi)^{2}} e^{-i \vec{\Delta}_{T} \cdot \vec{b}_{T}} F^{q}\left(x, \Delta_{T} ; S\right)=\mathcal{H}^{q}\left(x, \vec{b}_{T}^{2}\right)+\frac{\epsilon_{T}^{i j} b_{T}^{i} S_{T}^{j}}{M}\left(\mathcal{E}^{q}\left(x, \vec{b}_{T}^{2}\right)\right)^{\prime},
$$

where the derivative of $\mathcal{E}^{q}$ with respect to $\vec{b}_{T}^{2}$ enters. The correlator $\mathcal{F}^{q}$ has the following interpretation: it describes the distribution of unpolarized quarks carrying the longitudinal momentum fraction $x$ at a transverse position $\vec{b}_{T}$ inside a transversely polarized target.

If the second term on the r.h.s. in (6) is non-zero, $\mathcal{F}^{q}$ is not axially symmetric in $b$-space. In other words, the correlator is distorted. In fact, one can show in a model-independent way that for a nucleon target the correlator has a large distortion, where the effect for a quark flavor $q$ is proportional to the contribution of the corresponding flavor to the anomalous magnetic moment of the nucleon [1]. One may now speculate that this large distortion should have an observable effect. Indeed in [1] it was argued that it may be related to the Sivers function. An explicit form of the relation was obtained in Ref. [3] by considering the average transverse momentum of an unpolarized quark inside a transversely polarized target,

$$
\begin{aligned}
\left\langle k_{T}^{q, i}(x)\right\rangle_{U T} & =-\int d^{2} \vec{k}_{T} k_{T}^{i} \frac{\epsilon_{T}^{j k} k_{T}^{j} S_{T}^{k}}{M} f_{1 T}^{\perp q}\left(x, \vec{k}_{T}^{2}\right) \\
& =\int d^{2} \vec{b}_{T} \mathcal{I}^{q, i}\left(x, \vec{b}_{T}\right) \frac{\epsilon_{T}^{j k} b_{T}^{j} S_{T}^{k}}{M}\left(\mathcal{E}^{q}\left(x, \vec{b}_{T}^{2}\right)\right)^{\prime} .
\end{aligned}
$$

The result in (7) represents the first quantitative non-trivial relation between a GPD and a TMD. It also provides an intuitive explanation of the Sivers effect. (In this context we refer 
to $[1,2,3]$ where also the meaning of the object $\mathcal{I}^{q}$ is discussed.) However, the relation (7) is model-dependent. It was obtained in the framework of a simple spectator model of the nucleon, treated to lowest non-trivial order in perturbation theory [3]. On the other hand, the relation (7) is quite successful from a phenomenological point of view. Therefore, it makes sense to look for additional non-trivial relations, even if they turn out to be merely model-dependent.

\section{Model-independent considerations}

To get some guidance for further possible non-trivial relations the structures in the GPDand TMD-correlator can be compared $[4,7]$. This procedure was first used in the case of quark distributions [4], and later on extended to the gluon sector [7]. Besides the already mentioned trivial relations (called relations of first type in [7]), one finds the following list of non-trivial analogies/relations between GPDs and TMDs [7]:

- Relations of second type

$$
\begin{gathered}
f_{1 T}^{\perp q / g} \leftrightarrow-\left(\mathcal{E}^{q / g}\right)^{\prime}, \quad h_{1}^{\perp q} \leftrightarrow-\left(\mathcal{E}_{T}^{q}+2 \tilde{\mathcal{H}}_{T}^{q}\right)^{\prime}, \\
\left(h_{1 T}^{g}+\frac{\vec{k}_{T}^{2}}{2 M^{2}} h_{1 T}^{\perp g}\right) \leftrightarrow-2\left(\mathcal{H}_{T}^{g}-\frac{\vec{b}_{T}^{2}}{M^{2}} \Delta_{b} \tilde{\mathcal{H}}_{T}^{g}\right)^{\prime} .
\end{gathered}
$$

- Relations of third type

$$
h_{1 T}^{\perp q} \leftrightarrow 2\left(\tilde{\mathcal{H}}_{T}^{q}\right)^{\prime \prime}, \quad h_{1}^{\perp g} \leftrightarrow 2\left(\mathcal{E}_{T}^{g}+2 \tilde{\mathcal{H}}_{T}^{g}\right)^{\prime \prime} .
$$

- Relation of fourth type

$$
h_{1 T}^{\perp g} \leftrightarrow-4\left(\tilde{\mathcal{H}}_{T}^{g}\right)^{\prime \prime \prime} .
$$

To the best of our knowledge Eqs. (8)-(10) contain all possible non-trivial analogies/relations between leading twist GPDs and TMDs for quarks and gluons. Moreover, the method of Refs. $[4,7]$ only indicates which distributions may be related, but does not provide an explicit form of a relation.

\section{Model results}

In Ref. [7] we have studied two spectator models in order to find explicit forms of possible non-trivial relations: first, a scalar diquark spectator model of the nucleon; second, a quark target model treated in perturbative QCD, which also allows one to study relations between gluon distributions. We found it convenient to work with GPDs in momentum rather than impact parameter representation. The relations presented in the following involve moments of GPDs and TMDs, which (also for non-integer $n$ ) are defined according to

$$
\begin{aligned}
X^{(n)}(x) & =\frac{1}{2 M^{2}} \int d^{2} \vec{\Delta}_{T}\left(\frac{\vec{\Delta}_{T}^{2}}{2 M^{2}}\right)^{n-1} X\left(x, 0,-\frac{\vec{\Delta}_{T}^{2}}{(1-x)^{2}}\right), \\
Y^{(n)}(x) & =\int d^{2} \vec{k}_{T}\left(\frac{\vec{k}_{T}^{2}}{2 M^{2}}\right)^{n} Y\left(x, \vec{k}_{T}^{2}\right) .
\end{aligned}
$$


Taking as example the relation between the Sivers function and the GPD $E$, the relations of the second type have the form [7]

$$
f_{1 T}^{\perp q(n)}(x)=h_{2}(n) \frac{1}{1-x} E^{q(n)}(x), \quad(0 \leq n \leq 1) .
$$

The function $h_{2}(n)$ is different in the two models that we considered. We note that for all relations indicated in (8) a formula corresponding to (13) holds true. Evaluating (13) for $n=0$ and $n=1$ one recovers results presented earlier in Refs. [6,3]. In this context it is also worthwhile to mention that for $n=1 \mathrm{Eq}$. (13) is equivalent to the content of Eq. (7).

The model calculations provide the following explicit relation of third type [7],

$$
h_{1 T}^{\perp q(n)}(x)=h_{3}(n) \frac{1}{(1-x)^{2}} \tilde{H}_{T}^{q(n)}(x), \quad(0 \leq n \leq 1),
$$

and a corresponding formula for the gluon distributions in (9). In contrast to the previous case the function $h_{3}$ is the same in both models.

Eventually, we mention that the relation of fourth type in (10) is trivially satisfied in the quark target model, because to lowest non-trivial order in perturbation theory both the TMD $h_{1 T}^{\perp g}$ and the GPD $\tilde{H}_{T}^{g}$ vanish [7].

\section{Summary and discussion}

This note is dealing with the question if there exist non-trivial relations between GPDs on the one hand and TMDs on the other. On the basis of model-independent considerations one can distinguish between different types of possible non-trivial relations. It turns out that so far no model-independent non-trivial relations exist and it seems even unlikely that they can ever be established. However, many relations exist in the framework of simple spectator models, treated to lowest non-trivial order in perturbation theory. Once higher order diagrams are taken into consideration some of these relations are expected to break down [7]. Nevertheless, for instance the phenomenology and the predictive power of the loworder spectator model relation between the Sivers effect and the GPD $E$ works quite well. This is the only non-trivial relation which currently can be confronted with data. Additional input from both the experimental and theoretical side is required in order to further study all other relations between GPDs and TMDs. Future work will certainly shed more light on this interesting topic.

\section{References}

[1] M. Burkardt, Phys. Rev. D 66, 114005 (2002).

[2] M. Burkardt, Nucl. Phys. A 735, 185 (2004).

[3] M. Burkardt and D. S. Hwang, Phys. Rev. D 69, 074032 (2004).

[4] M. Diehl and P. Hägler, Eur. Phys. J. C 44, 87 (2005).

[5] M. Burkardt, Phys. Rev. D 72, 094020 (2005).

[6] Z. Lu and I. Schmidt, Phys. Rev. D 75, 073008 (2007).

[7] S. Meissner, A. Metz and K. Goeke, arXiv:hep-ph/0703176.

[8] Slides: http: //indico. cern. ch/contributionDisplay py? contribId=247\&sessionId=12\&conf Id=9499

[9] D. W. Sivers, Phys. Rev. D 41, 83 (1990); Phys. Rev. D 43, 261 (1991).

[10] D. Boer and P. J. Mulders, Phys. Rev. D 57, 5780 (1998). 